tables enable one to avoid a great deal of the multiplication of the ordinates by the proper cosines, which is inherent in the calculation of the coefficients. Further tables include the conversion from polar to rectangular coordinates (useful in passing from the two Fourier coefficients corresponding to a particular frequency to its amplitude and phase), Darwin's scheme for a large number of ordinates $(p=121)$, tables of squares, square roots, $(\sin \alpha) / \alpha$, and other tables of interest in harmonic analysis. The last quarter of the book is devoted to problems and examples in harmonic analysis, illustrating the use of the various preceding tables; also to problems in the analysis of hidden periodicities.

The present volume should prove very useful to anyone engaged in frequent harmonic analysis.

\title{
H. PORITSKY
}

Tables of Partitions. By Hansraj Gupta. Madras, Indian Mathematical Society, 1939. $5+81$ pp.

This work contains two tables. The first table gives the number $P(n)$ of unrestricted partitions of $n$ for $n \leqq 600$. The second table is a table of double entry giving the number $(n, m)$ of partitions of $n$ the least element of which is $m$ for $n \leqq 300$. As is explained in the introduction, the second table was calculated by the recursion formula $(n, m)=(n-m, m)+(n+1, m+1)$ together with certain special properties of the symbol $(n, m)$; a specimen calculation is given. The first table is a result of the second since $P(n)=(n+1,1)$. Adequate checks have been applied to insure accuracy. The introduction also contains summaries of certain papers by G. N. Watson and D. H. Lehmer on partitions.

The Indian Mathematical Society and the University of the Punjab are to be commended for bearing the cost of publication of this work. The table of unrestricted partitions has previously been published in two parts in the Proceedings of the London Mathematical Society. This table has been used by Chowla to disprove one case of a result conjectured by Ramanujan. This work should be very valuable to anyone interested in the subject of partitions.

\section{T. A. Pierce}

Elementary Mathematics from an Advanced Standpoint. Geometry. By Felix Klein. Translated from the third German edition by E. R. Hedrick and C. A. Noble. New York, Macmillan, 1939. 9+214 pp., 141 figs.

Another volume of Klein's masterful lectures is available in Eng- 